\title{
El capital informacional como condición de la inclusión digital: un análisis exploratorio
}

Informational capital as a condition of the digital inclusion: an exploratory analysis

Capital informacional como condição de inclusão digital: uma análise exploratória

\author{
Paola Espina Bocic \\ Escuela de Ciencias Jurídicas y Sociales \\ Universidad Viña del Mar, Chile \\ Jorge Gibert Galassi \\ Escuela de Negocios Internacionales \\ Universidad de Valparaíso, Chile \\ Autor referente: paola.espina@uvm.cl
}

Historia Editorial

Recibido: 28/04/2017

Aceptado: 04/10/2017

\section{RESUMEN}

El trabajo explora la siguiente hipótesis: el capital informacional de los "nativos digitales" es menor de lo esperado y, en ese sentido, su inclusión digital es débil. Las tecnologías de la información y las comunicaciones cumplen la labor de integración social de los ciudadanos del nuevo milenio, quienes estarían digitalmente "incluidos" cuando su capital informacional les permite participar igualitariamente de las oportunidades que brinda "esta" sociedad, al acceder a estas nuevas tecnologías para

mejorar sus condiciones de vida diarias y facilitar sus tareas en el mundo del trabajo. En nuestro estudio nos hemos centrado en la exploración del concepto de capital informacional desde una óptica operacional cuantitativa, que discute formulaciones anteriores $y$ sintetiza hallazgos recientes. Para ello, se aplicó una encuesta online a una muestra de universitarios chilenos de ambos sexos, entre 17 y 29 años, pertenecientes a programas de estudio en dos universidades, bajo el supuesto que por nivel educacional y edad son 
típicamente "ciudadanos digitales", esto es, nativos digitales "incluidos" en la sociedad de la información y el conocimiento. Las principales conclusiones apuntan a que existen habilidades digitales que poseen los estudiantes, como las aquí llamadas "protocolares", al mismo tiempo que se observa la carencia de algunas habilidades técnicas básicas para el mundo del trabajo. Finalmente, se constata que se podría aclarar más la potencialidad descriptiva del constructo "Capital informacional", actualizando las habilidades y competencias digitales para comprender mejor el significado de ser digitalmente alfabeto hoy, que es una condición para una inclusión digital efectiva.

Palabras clave: Capital informacional; Inclusión digital; Conocimiento digital; Ciudadanía informacional

\section{ABSTRACT}

The paper explores the following hypothesis: the informational capital of "digital natives" is less than expected and in that sense their digital inclusion is weak. ICTs fulfill the function of social integration of New Millennium citizens who would be digitally included when their informational capital allows them to participate equally in the opportunities offered by this society to access these new technologies to improve their daily living conditions, and facilitate their tasks in the world of work. In our study we have focused on the exploration of the concept of informational capital from a quantitative operational perspective that discusses previous formulations and synthesizes recent findings. To do this, an online survey was applied to a sample of Chilean university students of both sexes, between the 17 and 29 years of age, belonging to study programs in two universities under the assumption that by educational level and age they are typically digital citizens, i.e. digital natives "included" in the society of information and knowledge. The main conclusions point to the existence of digital skills that cross the students, as those called "protocol skills", but at the same time, the lack of some basic technical skills for the world of work. Finally, it is noted that "Informational capital" could be further clarified in terms of the descriptive potential of the construct, updating digital skills and competences to better understand the meaning of being digitally alphabet today, which is a condition for an effective digital inclusion.

Keywords: Informational capital; Digital inclusion; Digital knowledge; Informational citizenship

\section{RESUMO}

O trabalho explora a hipótese seguinte: O capital informacional dos "nativos digitais" é menor do que poderia se esperar e, nesse sentido, a sua inclusão digital é fraca. As tecnologias da informação e das comunicações exercem o papel de integração social dos cidadãos do novo milênio, os quais estariam "incluídos" digitalmente quando seu capital informacional permite-lhes participar em igualdade de condições das oportunidades que oferece "esta" sociedade, ao acessar às novas tecnologias para melhorar 
suas condições cotidianas de vida e facilitar seu desenvolvimento no mundo do trabalho. Nosso estudo foca na exploração do conceito de capital informacional desde uma perspectiva operacional quantitativa, discutindo formulações prévias e sintetizando descobertas recentes. Para isto, foi aplicado uma pesquisa online a uma amostra de universitários chilenos de ambos sexos, de entre 17 e 29 anos, pertencentes a programas de formação de duas universidades, partindo do suposto que, tanto por nível educativo quanto por idade, são tipicamente "cidadãos digitais", ou seja, nativos digitais "incluídos" na sociedade da informação e o conhecimento. As conclusões principais apontam à existência de habilidades digitais que os estudantes possuem, tais como as aqui chamadas de "protocolares", ao mesmo tempo que observa-se a carência de habilidades técnicas básicas para o mundo do trabalho. Finalmente, constata-se que poderia se clarificar mais a potencialidade descritiva do construto "Capital informacional", atualizando as habilidades e competências digitais para compreender melhor o significado de ser digitalmente alfabeto nos dias de hoje, o que é uma condição para uma efetiva inclusão digital.

Palavras chave: Capital informacional; Inclusão digital; Conhecimento digital; Cidadania informacional.

\section{Introducción}

a inclusión digital forma parte de los procesos contemporáneos de democratización. En efecto, si la democracia moderna incluye el acceso igualitario y el uso equitativo de las nuevas tecnologías de la información y las comunicaciones, entonces está cumpliendo la labor de integración social de todos los ciudadanos en la sociedad de la información. Así, un individuo digital "incluido" es aquel que participa igualitariamente de las oportunidades que brinda "esta" sociedad, que accede a estas nuevas tecnologías para mejorar sus condiciones de vida diarias, maximizar sus posibilidades de bienestar personal y facilitar sus tareas en el mundo del trabajo, además de adoptar una postura reflexiva sobre los usos e impactos de tales tecnologías. La inclusión digital es un componente de sobrevivencia en la llamada "sociedad informacional o del conocimiento" puesto que nos permite el acceso a una muy amplia gama de datos, informaciones, conocimientos, aprendizajes y redes. 
Sin embargo, la promesa democrática y los beneficios económicos de esta nueva Era han sido esquivos. Desde hace dos décadas (Castells, 1997) se postuló que la exclusión social es uno de los aspectos definitorios de la nueva sociedad en red, denominada "Sociedad de la Información". Eso significa que existen estructuras reproductoras de las desigualdades socio-económicas cuyo efecto no disminuye sino que se ve acrecentado por las nuevas modalidades de ensamblaje socio-tecnológicos. Además, los procesos de concentración de la riqueza y las estructuras tradicionales de larga data se mantienen en todo el mundo (Piketty, 2015)

Pero naturalmente, la sociedad no involuciona. La forma y el grado en que los individuos estén incluidos digitalmente en la sociedad tenderá a incrementarse, las industrias creativas aumentarán (Florida, 2002), el rol del conocimiento también (Paavola \& Hakkarainen, 2005) aun cuando las expansiones de las oportunidades vayan de la mano con la emergencia de restricciones y los nuevos ghettos sean ahora informacionales (Drahos \& Braithwaite, 2003).

Ahora bien, la literatura sobre las TICs y su rol en la sociedad actual ha tendido a centrarse en el mundo de la educación bajo la etiqueta, primero, de la alfabetización digital, y más recientemente, del sistema escolar. En el sistema de educación superior universitario, el uso de tecnologías y herramientas digitales se ha ampliado, produciendo entornos cuya característica es el ensamblaje entre la infraestructura tradicional (salas y otros espacios) y las plataformas online (Ellis \& Goodyear, 2010). Los estudios sobre los mecanismos de aprendizaje de los estudiantes en las universidades contemporáneas han explorado diversas facetas de la interacción caraa-cara y plataformas e-learning (Biggs, 2012; Larusson \& White, 2014).

Pero tal parece que las desigualdades sociales ampliamente definidas también tendrían expresión en las habilidades digitales en el mundo universitario. Entonces, desde el punto de vista de la política, la manera de atacar la inequidad digital es mediante estrategias - educacionales y de más amplio espectro - que aumenten las 
habilidades digitales de los nativos de la era internet, con el objetivo de eliminar las brechas digitales (ONU, 2015). Según Burin, Coccimiglio, González, \& Bulla (2016), los estudios de inclusión digital pueden contribuir a una nueva perspectiva sobre el rol docente y la instrucción escolar y académica en relación a las nuevas tecnologías, para generar un cambio social sustentable.

Entonces, cabe la pregunta: Los "Nativos Digitales”, ¿Qué tan incluidos están en la era internet? La evidencia internacional es que la brecha digital se manifiesta en la accesibilidad y el uso (Chinn \& Fairlie, 2004; Hilbert, 2013). Es esta última, la del uso, lo que nos interesa. Así, nuestro trabajo se focaliza en un grupo de estudiantes universitarios chilenos y centra la discusión a partir del concepto de capital informacional. Nuestra hipótesis es que el capital informacional de los "nativos digitales" es menor de lo esperado y, en ese sentido, su inclusión digital es débil.

\section{Capital informacional: una discusión conceptual sobre inclusión digital.}

La inclusión digital ha sido concebida en contraposición a la brecha digital y a la exclusión digital, que refiere a las desigualdades socio-económicas en el acceso y uso de las TICs. Al principio, la conceptualización era binaria y remitía a tener o no-tener acceso a una conexión a internet. A principios de los años noventa, la diferencia entre tener o no tener acceso era atribuida a una diferencia en el capital económico o meros ingresos familiares. Luego, hace 12-15 años atrás, la discusión acerca de las brechas se orientó al uso ó no-uso de internet y a los tipos y niveles de acceso. En los últimos años, la literatura ha ampliado el alcance de los conceptos de inclusión digital y brecha digital, incorporando los tópicos de habilidades, motivaciones y compromisos (engagement). (Zillien \& Hargittai, 2009; Witte \& Mannon, 2010).

Burin et al. (2016) citan a una diversidad de autores que plantean que en el centro de las competencias digitales existen habilidades digitales técnicas y operacionales (uso de dispositivos digitales), y habilidades cognitivas sobre búsqueda (cómo hallar 
información de forma eficiente), navegación (poder seguir información en forma dinámica y no secuencial), integración del conocimiento(ensamblar y sintetizar distintas fuentes), y evaluación del contenido (pensar de forma crítica sobre fuentes y contenidos) (Bawden, 2008; Lankshear \& Knobel, 2008; Van Deursen \& VanDijk, 2014).

Así, el acceso físico a computadores, móviles e internet dio paso a la discusión sobre alfabetización tecnológica y digital, contenidos, usos, redes y costos asociados (Moyo, 2009). También y crecientemente ha surgido la preocupación por la falta de interés en conectarse a internet, más visible en ciertos grupos (adultos mayores y otros) que no logran experimentar las ventajas inmediatas o estratégicas de la participación en el mundo digital.

La prospectiva de la teoría de la inclusión digital avanza hacia una conceptualización compleja que debería "sumar" de algún modo cuatro dimensiones:

Acceso (1): Es decir, la posesión de un buen equipo móvil, disponibilidad relativamente permanente de banda ancha / wi-fi de calidad, etc.

Habilidades (2): Las destrezas técnicas, las sociales y de protocolos de la comunicación válida, las habilidades críticas en torno a las fuentes, y las habilidades creativas, como las típicas de editar un video o construir una página web.

Motivacional (3): La existencia de motivaciones sociales (como ser parte del grupo) y de motivaciones personales.

Compromisos (4): La manera, grado y modos en que estamos involucrados y orientamos nuestra práctica online hacia un compromiso cívico-político, social, educacional, económico o cultural.

En nuestro trabajo nos hemos concentrado en las dos primeras dimensiones, especialmente en la segunda (habilidades), desde una óptica metodológica particular, cual es la de hacer equivalente el acceso y las habilidades al concepto de capital informacional. En otras palabras, nuestro interés se centró en la exploración de las 
posibilidades del constructo, dejando para una discusión posterior si acaso el capital informacional puede ser visto como un componente del fenómeno más amplio de la inclusión digital o bien es un requisito o conditio sine qua non de ella. Nuestra idea fue aproximarnos cuantitativamente a la comprensión del capital informacional de un grupo hipotéticamente de alto consumo digital, cual es, los estudiantes universitarios.

El Capital Informacional es un derivado del concepto de Capital Cultural, de la teoría del campo social de Pierre Bourdieu (2011). El capital cultural es el stock de conocimiento ampliamente definido, acumulado por un individuo a lo largo de su vida, que usa para mantener o alcanzar posiciones de mayor status y poder en la jerarquía del mundo social, e incluye su educación formal e informal, así como las maneras de comportarse y vincularse con el mundo material. El capital cultural aparece bajo tres formas: 1) objetivado, son los soportes materiales del capital cultural (objetos, aparatos); 2) institucionalizado, básicamente los certificados de educación formal y otros tipos de ritos de legitimación; y 3) incorporado, la incorporación en el cuerpo (gestos, rutinas) de la cultura mediante la educación informal, principalmente de la familia, y que se despliega bajo la modalidad de performances y estrategias de exhibición en el mundo social (Bourdieu, 2011). Según Drabowicz (2017), en consonancia con el trabajo de Prieur \& Savage (2013), la conceptualización del capital cultural inscrito en la tradición Bourdieusiana permite afirmar que para que éste adquiera su pleno significado, deberíamos llamarlo capital informacional, pues captura mejor las nuevas tendencias de desarrollo social en la Era internet.

El capital informacional también posee otra vía de entrada, como la ofrecida por (Hamelink, 2001). En su propuesta, el Capital Informacional es definido como la capacidad financiera para pagar la utilización de redes electrónicas y servicios de información, la habilidad técnica para manejar las infraestructuras de estas redes, la capacidad intelectual para filtrar y evaluar la información y la habilidad para aplicar la información a situaciones sociales (p.91). 
Podemos definir cuatro componentes del capital informacional de un individuo: a) la posibilidad de pagar la tenencia de un dispositivo (fijo o móvil) y el acceso a conexión, b) la habilidad técnica para administrar el uso de la conexión, c) la capacidad para evaluar y filtrar la información, y d) la motivación para buscar información y para usar esa información en la sociedad para su propio beneficio.

Otra manera de enfrentar el desafío de conceptualizar el capital informacional es el materializado por Van Dijk (2005), quien ha centrado la atención en los tipos de acceso a las tecnologías digitales, desde una perspectiva procesual. Marí \& Sierra (2008) enfatizan otro aspecto: el capital informacional se vería como el proceso de generación y apropiación de la información por parte de los actores sociales, políticos y gubernamentales. Alva de la Selva (2016), indaga sobre la posibilidad de un indicador cualitativo de "Capital Informacional". A partir de un trabajo de campo en Ciudad de México, se desprende que el desarrollo de las competencias y habilidades técnicas que los jóvenes han adquirido son mucho más cercanas a sus motivaciones y necesidades que lo originalmente previsto.

En base a la discusión anterior, podemos afirmar que la controversia conceptual está en pleno desarrollo. Un aspecto importante, de modo de no meramente repetir los objetivos de las agendas de investigación de los países centrales, consiste en la exploración inductiva, a partir de las propias experiencias de los actores involucrados en los contextos situados de la acción. A continuación, se presenta nuestra propuesta exploratoria de medición del capital informacional, en base al Tabla 1, inspirada teóricamente en las visiones más robustas de Bourdieu y Hamelink. 


\section{Tabla 1}

Dos teorías y una propuesta de definición estructural del concepto de capital informacional.

\begin{tabular}{|c|c|c|c|}
\hline $\begin{array}{l}\text { Bourdieu (1979) } \\
\text { Capital cultural }\end{array}$ & $\begin{array}{l}\text { Hamelink (2001) } \\
\text { Capital informacional }\end{array}$ & $\begin{array}{l}\text { Espina \& Gibert } \\
\text { Capital informacional }\end{array}$ & $\begin{array}{l}\text { Figuras }(F) \\
\text { y tablas }(T)\end{array}$ \\
\hline $\begin{array}{l}\text { Objetivado } \\
\text { (los aparatos y } \\
\text { conexiones) }\end{array}$ & $\begin{array}{l}\text { Pagar la tenencia de un } \\
\text { dispositivo (fijo o móvil) y } \\
\text { el acceso a conexión }\end{array}$ & Tenencia y acceso & $\mathrm{F}-1$ \\
\hline $\begin{array}{l}\text { Institucionalizado } \\
\text { (legitimaciones } \\
\text { convencionales) }\end{array}$ & $\begin{array}{l}\text { Capacidad para evaluar } \\
\text { y filtrar la información }\end{array}$ & $\begin{array}{l}\text { Habilidades } \\
\text { protocolares }\end{array}$ & $\begin{array}{l}F-2 \\
F-3 \\
F-4\end{array}$ \\
\hline $\begin{array}{l}\text { Incorporado } \\
\text { (educación } \\
\text { formal) }\end{array}$ & $\begin{array}{l}\text { Administrar el uso de la } \\
\text { conexión }\end{array}$ & Habilidades técnicas & $\begin{array}{l}\text { T-2 } \\
\text { F-5 }\end{array}$ \\
\hline $\begin{array}{l}\text { Incorporado } \\
\text { (educación } \\
\text { informal) }\end{array}$ & $\begin{array}{l}\text { Motivación para buscar } \\
\text { información y usarla en } \\
\text { su propio beneficio }\end{array}$ & Rutinas sociales & $\begin{array}{l}\text { T-3 } \\
\text { F-7 }\end{array}$ \\
\hline & propia. Las teorías a la & on $B$ & (2011). L \\
\hline
\end{tabular}

En nuestra propuesta asimilamos teóricamente la discusión de la siguiente manera: 1) como no hay capital sin valor (patrimonial, económico o material), entonces el capital informacional posee un soporte material, esto es, tenencia y acceso ilimitado; 2) el constructo opera también como moneda de cambio o mecanismo institucionalizado (legitimado) que funciona optimizando el uso del tiempo para la transacción entre proveedores y usuarios de internet bajo estrategias protocolares, protocolos establecidos del modo y herramientas "correctas-adecuadas" de búsqueda y uso de información; 3) el capital informacional apunta igualmente al dominio de técnicas indispensables en el mundo laboral y en el cotidiano, que facilitan la vida virtual y rentabilizan la educación y capacitación formal; y 4) el uso predeterminado de aplicaciones en internet y el tiempo ocupado en ellas configura un modo de vida o un conjunto de rutinas sociales en los individuos, resultado de la adquisición espontánea en el marco de las interacciones con sus pares (etarios, de clase social u oficios).

Cada componente se tradujo a dos o más preguntas que permitieron la exploración cuantitativa. 


\section{Metodología}

El objetivo del trabajo fue materializar una aproximación empírica al concepto de capital informacional, entendido como un conjunto de propiedades adquiridas por los individuos en virtud de su condición de "nativos digitales". Para ello, se analizó la literatura y se postuló una definición operacional (estructural) compuesta de cuatro componentes, donde cada uno de ellos se desagregó en dimensiones y sus respectivos indicadores, los que fueron descritos (medidos) en el estudio. Esta aproximación empírica permitió, a su vez, discutir preliminarmente la conexión entre la posesión de un cierto grado de capital informacional y la auto-percepción por parte de la muestra de jóvenes universitarios chilenos de estar digitalmente incluidos en la sociedad de la información. En suma, permitió discutir si la magnitud del capital informacional es una condición del grado de inclusión digital auto-percibido.

El diseño de investigación es no-experimental transaccional, de tipo exploratorio descriptivo. Se usó un muestreo no probabilístico, intencionado hacia los jóvenes universitarios, debido a que el interés del estudio no es caracterizar a la población juvenil en su conjunto, sino realizar una aproximación cuantitativa de carácter exploratorio al concepto de capital informacional. Ello admite suponer que las unidades de análisis son los individuos intrínsecamente considerados "nativos digitales", y por tanto, hipotéticamente poseedores de un alto capital informacional. Ello permitiría una aproximación rápida al test propuesto, es decir, la verificación de la hipótesis del trabajo: el capital informacional de los "nativos digitales" es menor de lo esperado y, en ese sentido, su inclusión digital es débil. El tamaño $(n=168)$ es suficientemente voluminoso como para extraer algunas ideas generales e identificar algunas hipótesis para futuros estudios que permitan validar nuestra propuesta de medición del capital informacional. La recolección de la información se hizo desarrollando una encuesta online, que incluía el consentimiento informado, ésta fue aplicada a una muestra de jóvenes universitarios chilenos de ambos sexos, entre 17 y 29 años, pertenecientes a 
programas de estudio en dos universidades (una pública, la Universidad de Valparaíso y la otra privada, la Universidad Viña del Mar). Los datos fueron recogidos durante la primera semana de abril del 2017. Para el análisis se utilizó el paquete estadístico SPSS versión 15.0.

\section{Resultados}

Las características de la muestra son las siguientes: Total de individuos que responden, 168; 120 mujeres (71,4\%) y 48 hombres (28,6 \%); 72 estudiantes de la Universidad de Valparaíso (pública) y 96 estudiantes de la Universidad Viña del Mar (privada). De los 168 estudiantes, sólo 1 no posee un celular personal o propio. La clasificación según rango de edades es: a) 17-19 años (34,5\%, 58 estudiantes); 2) 2021 años (35,1\%, 59 estudiantes); y c) 22-29 años (30,4\%, 51 estudiantes).

\section{Primer componente: Tenencia y acceso}

Todos poseen un teléfono celular propio. Se observa que sólo el 15\% de los jóvenes no accede a conexión de internet de un modo permanente y 2 de cada 3 tiene un plan pagado (figura 1).

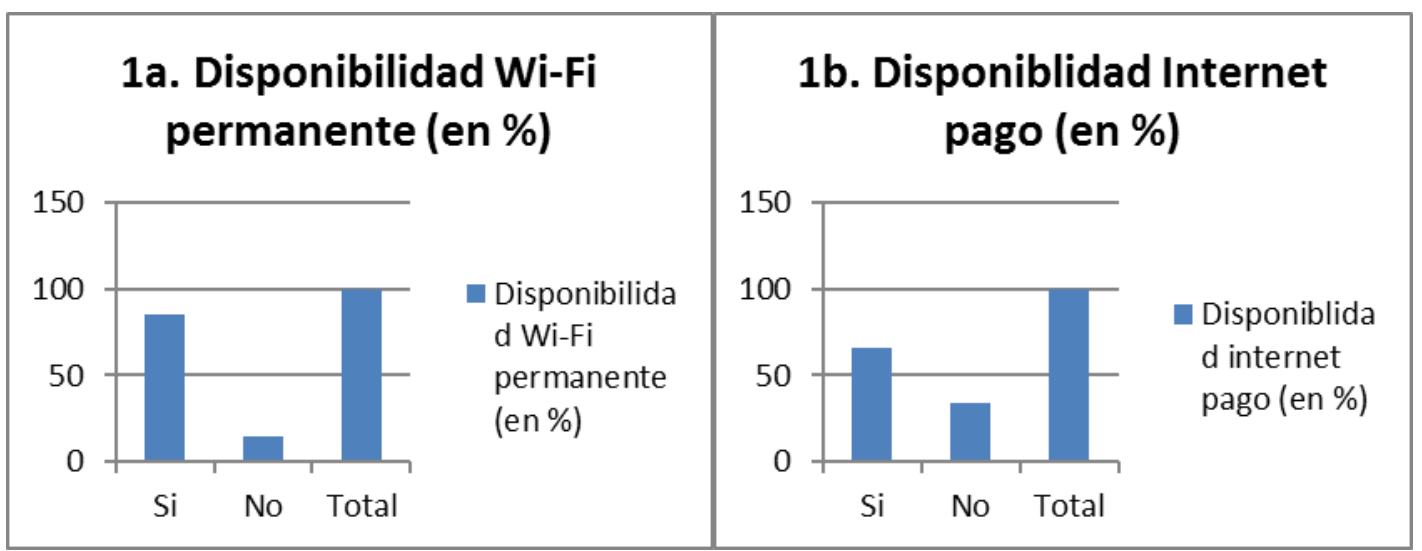

Figura 1. Población con disponibilidad de internet en teléfono celular. 
Tanto la Figura 1a como el 1b nos ilustran acerca del acceso como componente material del concepto de capital informacional y como condición de la inclusión digital. Precisamente dado que hoy la conexión es masiva en muchos países del mundo periférico, como Chile, ello nos alerta de la creciente importancia de los otros componentes del capital informacional.

\section{Segundo componente: Habilidades protocolares.}

Hemos operacionalizado las habilidades protocolares como aquellas acciones vinculadas a la optimización de las búsquedas de información en la web, mediante hábitos (protocolos) eficientes. Generamos tres dimensiones con varios indicadores para cada una.

La primera dimensión es el uso de la sintaxis de Google. Como se aprecia en la figura 2, el conocimiento de herramientas básicas en el uso de la sintaxis de Google para la búsqueda de información posee una distribución dispar. Lo más usado por los jóvenes es escribir la dirección URL del sitio que buscan, con un 47\%; mientras que la menos usada es la creación de alertas, con un 11\%.

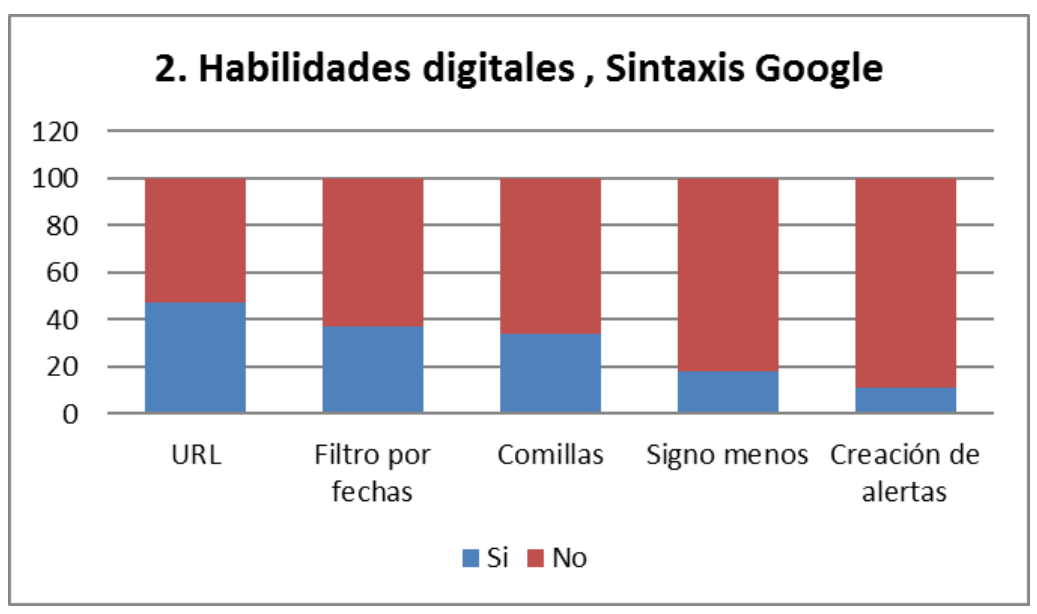

Figura 2. Porcentaje de la población con habilidades digitales, Sintaxis Google.

La segunda dimensión es el modus operandi para evaluar y filtrar las páginas web según calidad percibida e información que provee (figura 3). En relación con la 
evaluación y filtrado de información es notoria la atención que muestran sobre el tipo de documento. La autoridad de la fuente y la antigüedad/actualización (mayor antigüedad y actualizaciones frecuentes) del sitio se posicionan en un segundo lugar. Dos de cada tres estudiantes se fija en usar información que también esté repetida (o citada) en otros sitios de igual o mayor prestigio. Finalmente, sorprende que sólo un tercio revisa más allá de las primeras 10 sugerencias que arroja Google.

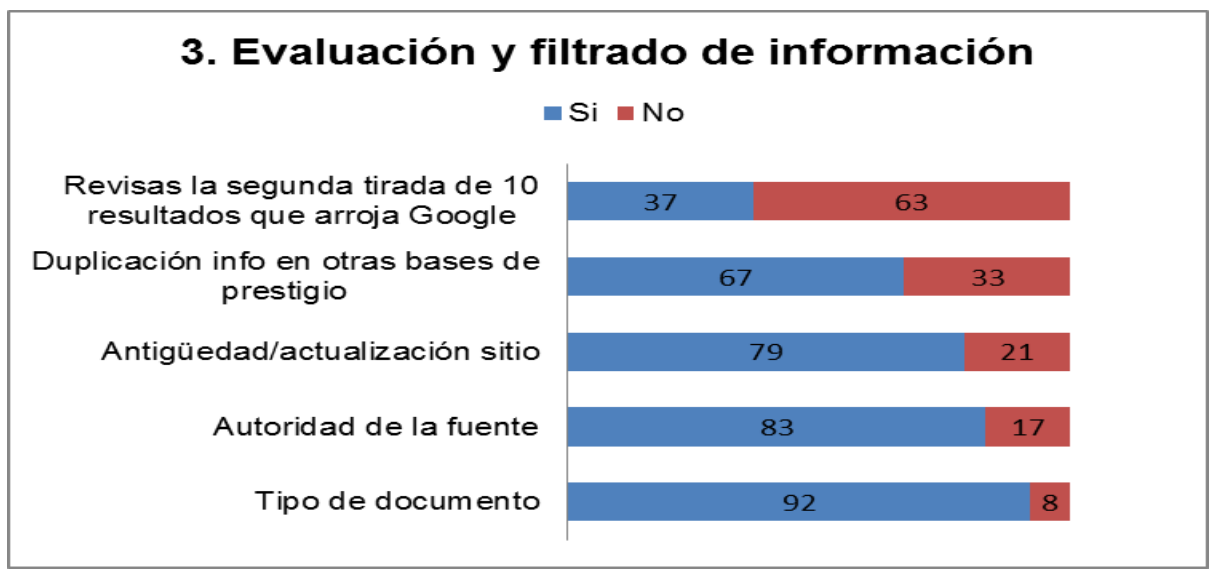

Figura 3. Porcentaje de la población, según tipo de evaluación y filtrado de información que usa.

La tercera dimensión consiste en el conjunto de herramientas de trabajo usadas habitualmente por los usuarios (figura 4). En el contexto de su vida académica, las herramientas digitales más usadas son Google académico, los sitios oficiales y las revistas científicas. Llama la atención el alto uso de revistas científicas, un $85 \%$. También es notorio el bajo uso de Wikipedia, un 40\%. El comportamiento lógico se ve desafiado por la realidad del proceso de enseñanza-aprendizaje, ya que se podría suponer que la mayoría utiliza para un trabajo académico Wikipedia, dado el carácter de "entrada" a un tema desconocido que define a toda enciclopedia, pero es precisamente lo más complejo lo que más se usa, el Google académico y las revistas de especialidad. 


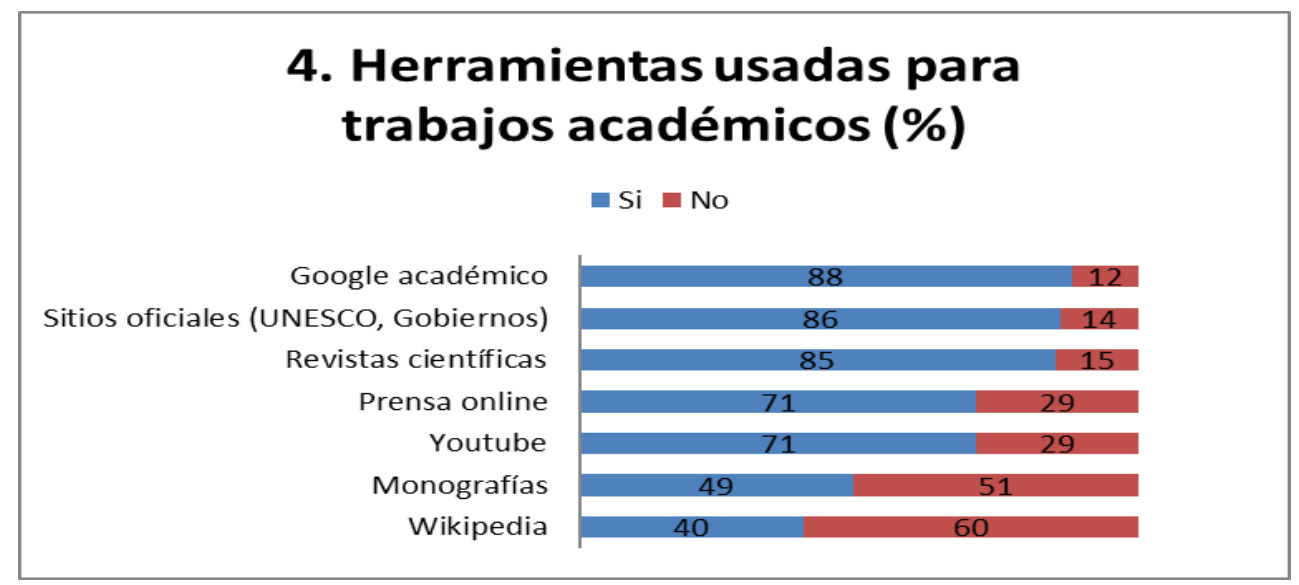

Figura 4. Herramientas usadas por la población para trabajos académicos.

\section{Tercer componente: Habilidades técnicas.}

Las habilidades técnicas las hemos definido de un modo grueso, como aquellas indispensables para cultivar y beneficiarse del intercambio digital. Generamos dos dimensiones con varios indicadores para cada una.

Una primera dimensión es el conjunto de habilidades de ofimática, es decir, el dominio de tres tipos de archivos para crear y compartir información en el marco de la vida laboral, comúnmente usados a diario en el mundo digital.

El dominio de ofimática (Word, Excel y PowerPoint) se aprecia en la tabla 2. La mitad de los jóvenes se autoevalúa en un nivel de dominio intermedio. Un tercio de los usuarios de Word y PowerPoint piensan que poseen un dominio avanzado de la herramienta, mientras que sólo un $12 \%$ se considera un usuario con dominio avanzado de Excel. 


\section{Tabla 2}

Dominio de ofimática

\begin{tabular}{lllll}
\hline & \multicolumn{4}{l}{ DOMINIO DE HERRAMIENTAS OFIMÁTICAS } \\
\hline & Básico & Intermedio & Avanzado & TOTAL \\
& $\%$ & $\%$ & $\%$ & \\
\hline WORD & 11 & 57 & 32 & 100 \\
POWERPOINT & 13 & 56 & 31 & 100 \\
EXCEL & 36 & 42 & 12 & 100 \\
TOTAL & $101(20 \%)$ & $260(52 \%)$ & $126(25 \%)$ & $487(100 \%)$ \\
FRECUENCIAS (\%) & & & &
\end{tabular}

Nota: De un $\mathrm{N}$ muestral de 168 casos, el total de respuestas teóricas es 504.17 casos corresponden a estudiantes que no usan Excel.

La segunda dimensión de este tercer componente es un conjunto de competencias digitales menos usuales en el mundo laboral pero igualmente masivas, definidas mediante un gradiente de complejidad que va desde lo más simple, instalar una aplicación, a operaciones más complejas como crear páginas web.

Respecto de éstas competencias digitales (figura 5), hay un gradiente de complejidad que exhiben los jóvenes al pasar de la instalación de aplicaciones, donde el $80 \%$ declara la competencia de realizar esa acción, a la creación de páginas web, donde menos del $20 \%$ posee esa competencia.

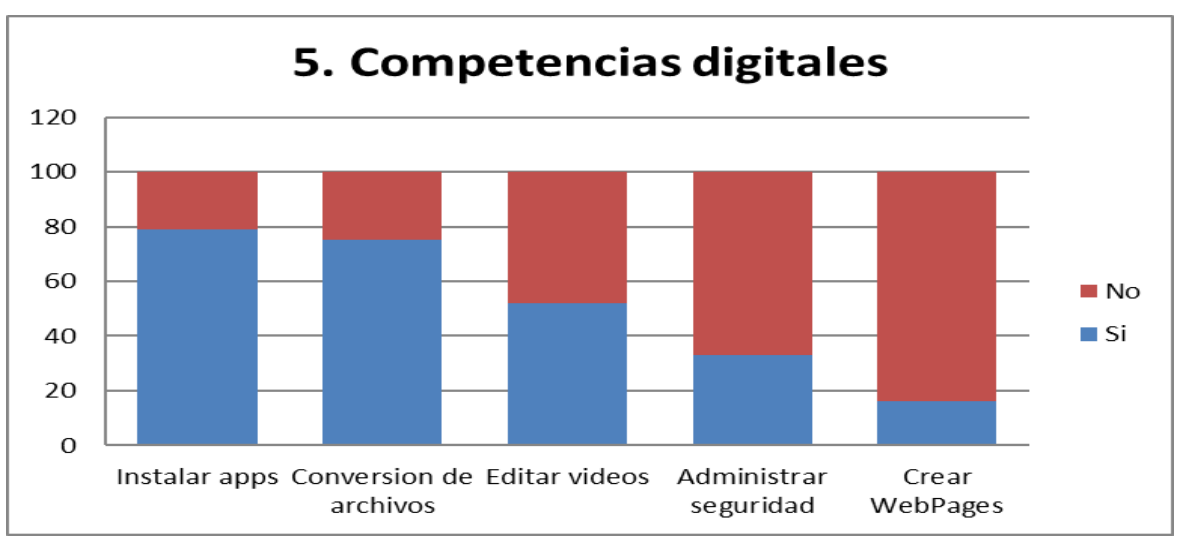

Figura 5. Competencias digitales.

\section{Cuarto componente: Rutinas sociales.}

Nuestro cuarto componente se define como el uso rutinario de determinadas aplicaciones en internet, lo que sumado al tiempo ocupado en ellas, configuran un 
modo de vida o un conjunto de rutinas sociales en los individuos. Tales rutinas son el resultado de la adquisición espontánea en el marco de las interacciones con sus pares (etarios, de clase social u oficios).

Las rutinas se componen en nuestra exploración de tres dimensiones: 1) las aplicaciones sociales; 2) el tiempo de uso; y, 3) el tipo de uso o la orientación predominante de uso, laboral o entretenimiento.

En relación con el uso de aplicaciones, se observa que existe un alto consumo de aplicaciones sociales más orientadas a la entretención, mientras que las aplicaciones laborales como Linkedln solo la usa el $6 \%$ de los jóvenes. Twitter que es concebido como una aplicación de intereses especiales u orientado hacia compromisos cívicos o culturales es usada por el $22 \%$ de los jóvenes. Slideshare, más bien de uso educativo, sólo es usada por el $11 \%$. Spotify, de tipo recreativo, es usado por el $45 \%$ de los jóvenes.

\section{Tabla 3}

Uso de aplicaciones por día.

\begin{tabular}{|c|c|c|c|c|c|c|}
\hline & \multicolumn{6}{|c|}{$\%$ de jóvenes que usa } \\
\hline & $\begin{array}{l}\text { Lo usa } \\
\%\end{array}$ & $\begin{array}{l}\text { Menos } \\
\text { hora \% }\end{array}$ & de & 1 & $\begin{array}{llll}\text { Entre } & 3 & \text { y } & 4 \\
\text { horas } \% & & \end{array}$ & $\begin{array}{l}\text { No Ic } \\
\text { usa \% }\end{array}$ \\
\hline GOOGLE & 100 & 43 & & & 15 & 0 \\
\hline WHATSAPP & 98 & 5 & & & 68 & 2 \\
\hline FACEBOOK & 97 & 17 & & & 38 & 3 \\
\hline YOUTUBE & 96 & 29 & & & 19 & 4 \\
\hline INSTAGRAM & 82 & 15 & & & 31 & 18 \\
\hline
\end{tabular}

La presunción de que la cantidad de horas de uso de aplicaciones variaba según la etapa de sus vidas académicas no se confirmó con la fuerza esperada. Sólo existe una ligera disminución de jóvenes que usan intensamente WhatsApp cuando se encuentran en la etapa terminal de sus carreras, mientras que sube el porcentaje de jóvenes en la etapa terminal que usan Youtube en períodos de menos de 1 hora, duplicándose probablemente para fines educacionales (tabla 3). En resumen, existe 
una variación mínima en el porcentaje de jóvenes en diversas etapas de su carrera académica respecto al uso de los medios sociales (figura 6).

Por cierto, una actividad rutinaria cuando los jóvenes están conectados en los social media es el posteo de información que es considerada "de interés" por parte de los usuarios. El 57\% reenvía toda la información que consideran "interesante".

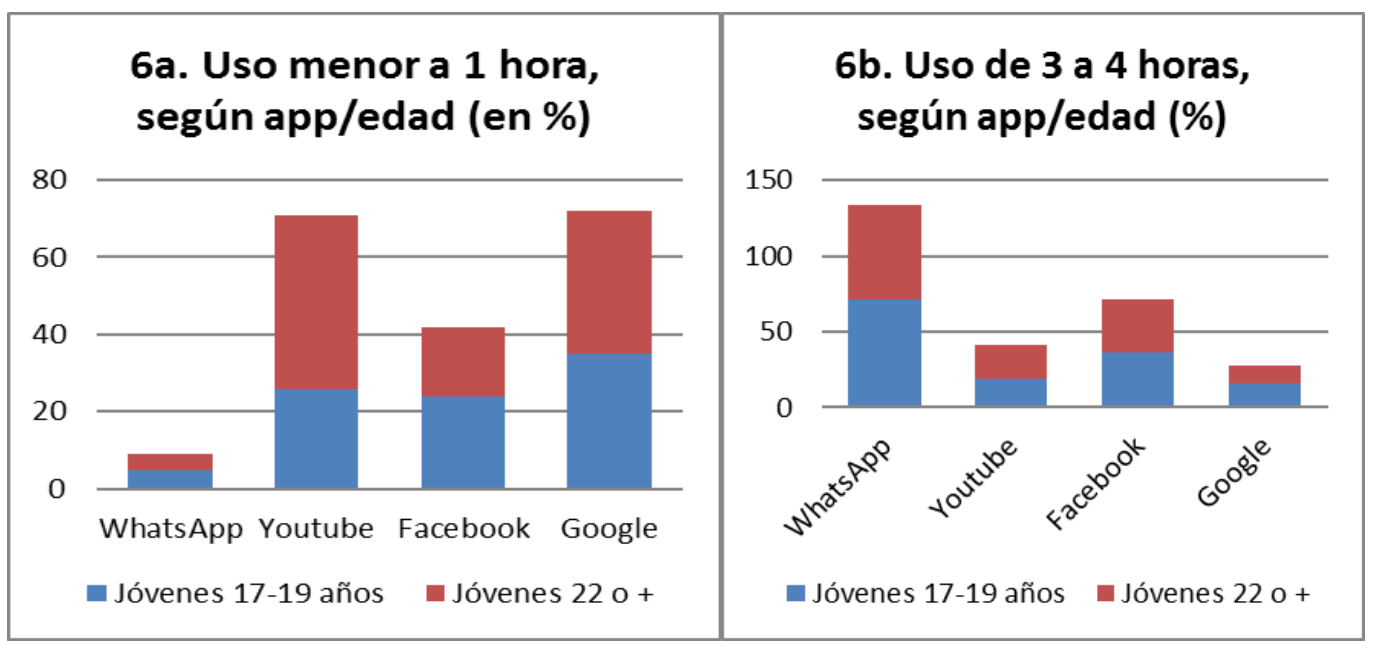

Figura 6. Horas de uso de aplicaciones según edad.

En términos de las horas de uso exclusivo al día, la categoría de entretención supera en 10 puntos a la categoría laboral o educacional (figura 7). Sin embargo, es plausible que ello se deba a un consumo total que puede ser bajo (en ambos ítems, laboraleducacional y entretención) o medio o alto (también en ambos ítems). Con ello de algún modo se rompe el prejuicio adulto que los jóvenes básicamente usan el celular para objetivos de entretenimiento. 


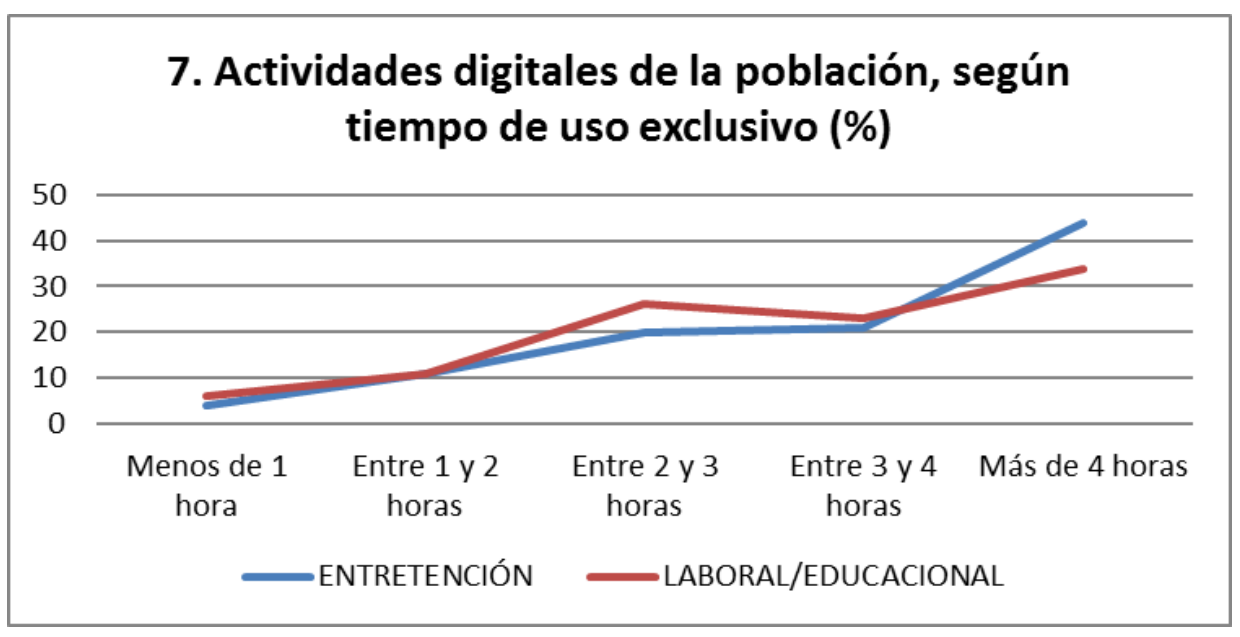

Figura 7. Actividades digitales de la población, según tiempo de uso exclusivo.

La variable sexo, en el figura 8 , se comporta de modo discriminante, asignando una cantidad de horas dedicadas exclusivamente a actividades lúdicas al sexo masculino, quizás producto de las rutinas de videojuegos. La dispersión es bastante similar entre hombres y mujeres cuando desarrollan actividades educativas, pero hay mayor dispersión entre las mujeres en actividades de entretención.

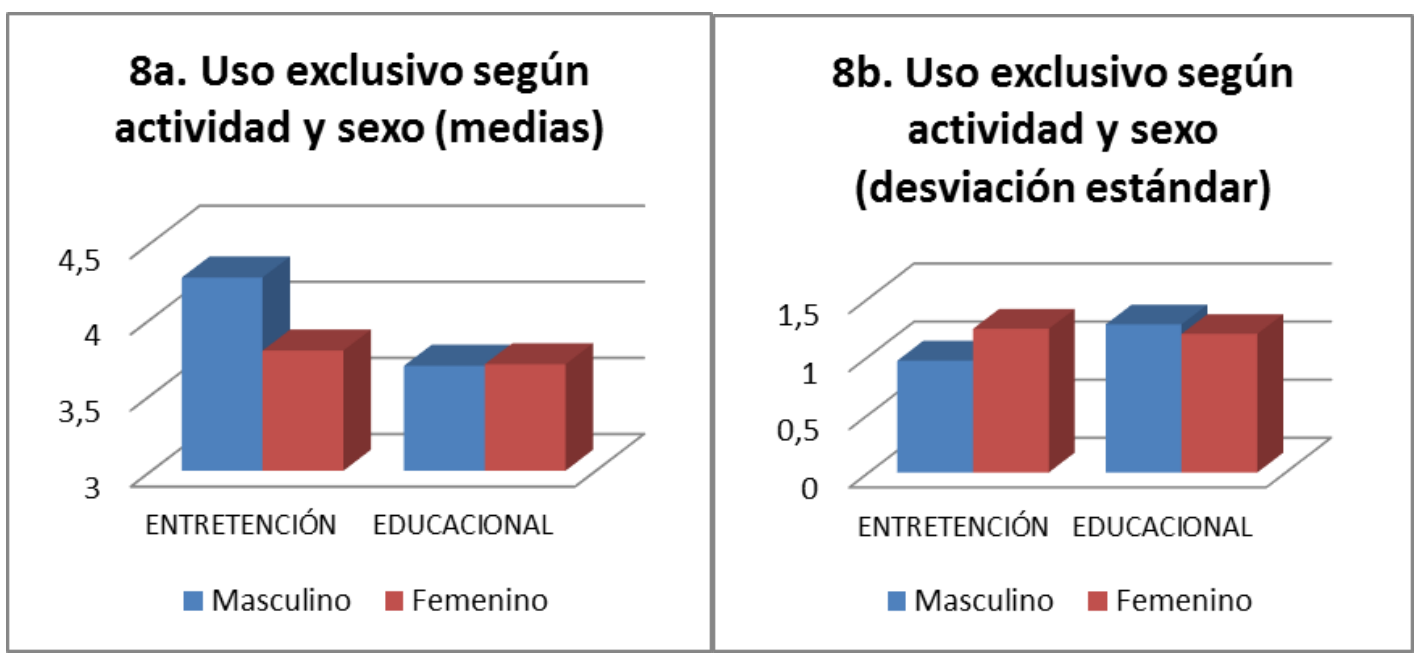

Figura 8. Uso exclusivo en horas, según actividad y sexo.

Siempre los jóvenes, sin importar la edad, ocupan más horas en actividades lúdicas en vez de en actividades educacionales. Sin embargo, la cantidad de horas dedicadas a la entretención forman una curva, donde los jóvenes de 17-19 y 22-29 ocupan menos 
horas que los jóvenes de 20-21. Es decir, en el período intermedio de sus vidas académicas dedican más tiempo promedio a actividades de entretención online, mientras que al inicio y al final, menos. Por otro lado, a medida que aumenta la edad, se forma una línea recta ascendente, pues el número de horas promedio usadas en educación y/o laboral aumenta (figura 9). Esto es consistente con las desviaciones estándar que cada grupo ostenta respecto del uso exclusivo de tiempo en ambas actividades, que varía mínimamente.

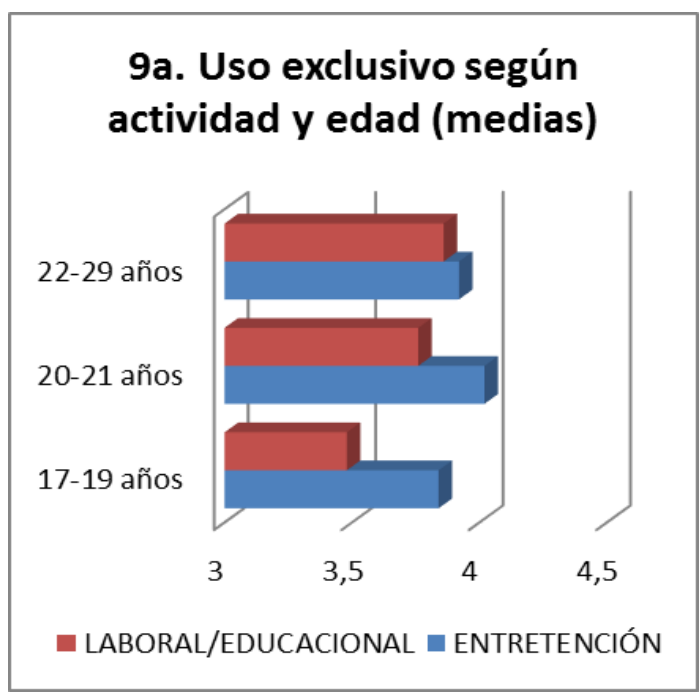

Figura 9. Uso exclusivo en horas, según actividad y edad.

Se ha construido un panorama del capital informacional poseído por los estudiantes. El razonamiento es que este panorama debería ser relativamente equivalente con la magnitud en que los estudiantes se sienten digitalmente incluidos. La inclusión digital sólo fue explorada mediante dos reactivos subjetivos de pertenencia, a través de la pregunta por la auto-percepción de ser nativo digital y la auto-percepción de ser ciudadano del mundo digital.

Finalmente, entonces, al preguntar por su identidad digital y su sentido de pertenencia a la sociedad de la información y el conocimiento, ellos manifiestan una brecha, que hace sospechar que efectivamente existe una impresión de déficit de habilidades digitales o que el mundo digital - aunque los define plenamente - posee ciertos rasgos 
amenazantes o de sentido de exclusión (figura10). El intervalo 52-67\% es la medida de magnitud encontrada en los estudiantes, su propia definición de cuánto se sienten parte del mundo contemporáneo en términos de inclusión digital.



Figura 10. Identidad socio-digital.

Una mayoría discreta de los estudiantes se considera un ciudadano de la sociedad de la información y el conocimiento, sin distinción de sexo. El porcentaje baja ligeramente, más en las mujeres, al responder la pregunta de si acaso se considera un nativo digital (figura 11).

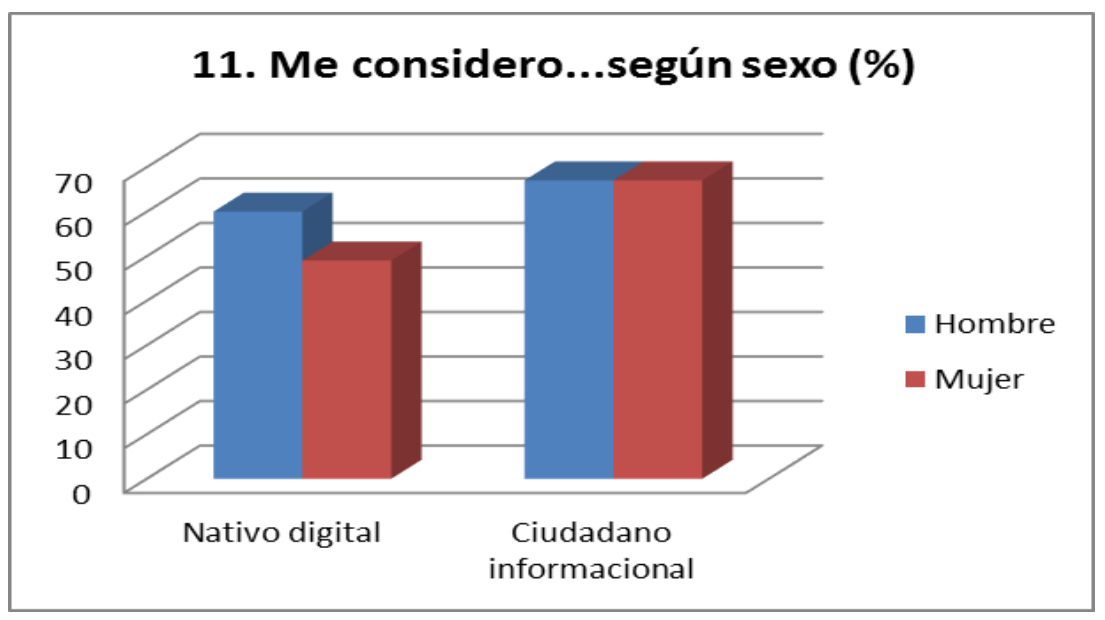

Figura 11. Me considero Nativo digital/Ciudadano de la sociedad de la información y el conocimiento, según sexo.

La auto-percepción de ser nativo digital es relativamente estable según la edad de los encuestados (figura 12). Sin embargo, a medida que aumenta la edad, la calidad de 
miembro o ciudadano de la sociedad de la información y el conocimiento decae progresivamente, distanciándose en más de $10 \%$ la diferencia entre quienes cursan los primeros dos años de universidad respecto de los estudiantes de últimos años.

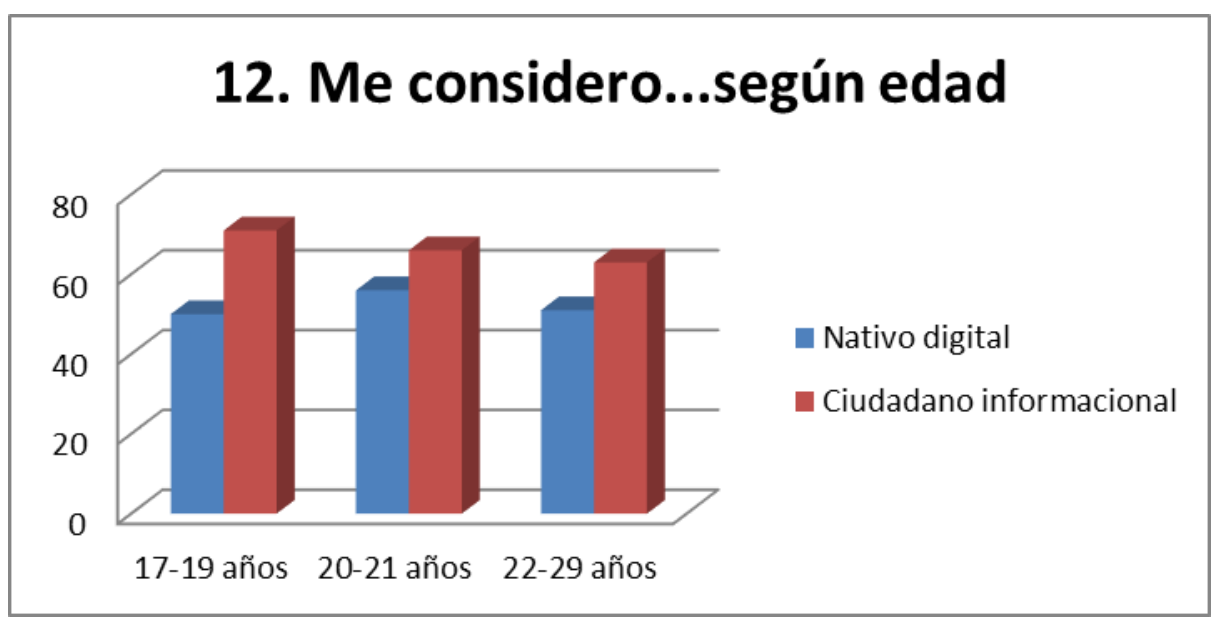

Figura 12. Me considero Nativo digital/Ciudadano de la sociedad de la información y el conocimiento, según edad.

La pertenencia a un tipo de universidad invierte las preferencias respecto a considerarse miembro de la sociedad de la información y el conocimiento o bien considerarse un nativo digital (figura 13). Mientras los estudiantes de la universidad pública se sienten nativos digitales en mayor proporción que los estudiantes de la universidad privada, éstos últimos se sienten ciudadanos de la sociedad de la información en mayor medida que sus pares de universidad pública. 


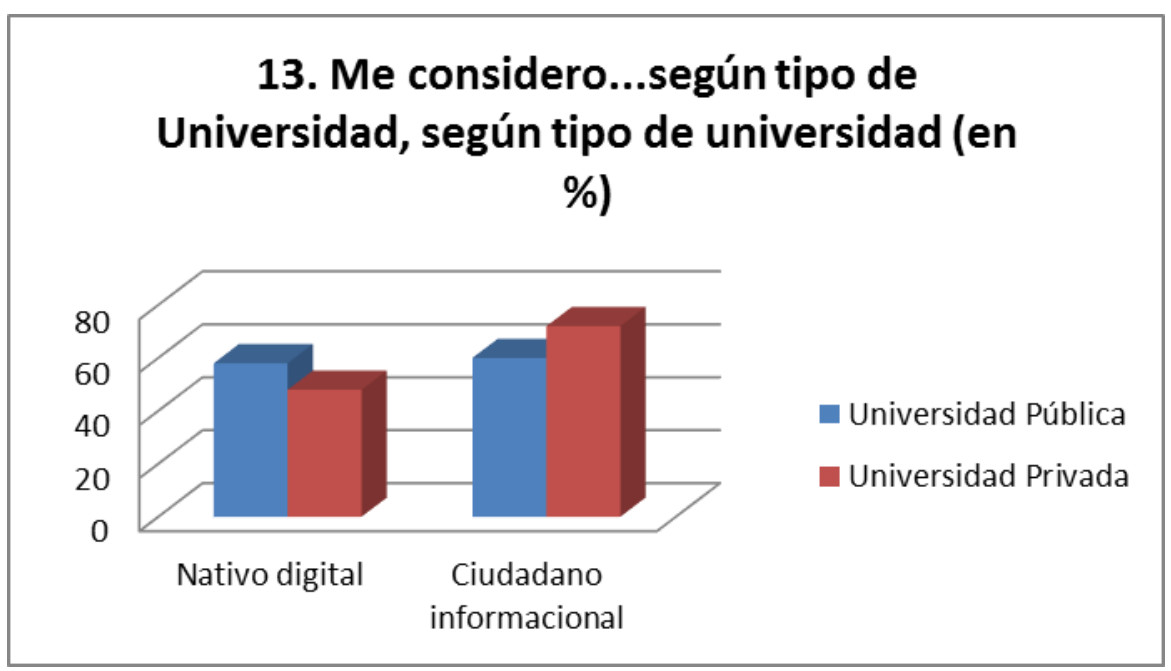

Figura 13. Me considero Nativo digital/Ciudadano de la sociedad de la información y el conocimiento, según tipo de Universidad.

En base a estos resultados, se presentan la discusión y una conclusión preliminar.

\section{Discusión de los resultados}

Este estudio concibió la inclusión digital de una manera subjetiva, según la autopercepción de los individuos. El supuesto fue que, en función de la reflexión y autoevaluación que los estudiantes hacen de su propio capital informacional, a medida que respondían el cuestionario online, se configuró una apreciación respecto del grado en que están incluidos digitalmente en la sociedad de la información y el conocimiento. Ello repercutió también en las respuestas sobre su identidad digital.

Los resultados permiten constatar que efectivamente los componentes objetivos de acceso al mundo digital ya no describen las desigualdades como antes, pues la gran mayoría posee móvil con conexión a internet. Así, los resultados avalan la idea que los componentes claves del capital informacional son los de uso, que comprenden tanto las habilidades (protocolares y técnicas) así como las rutinas sociales implicadas.

Sobre estas últimas, uno de los "mitos" que el estudio cuestiona es la relevancia del móvil para fines exclusivamente de entretención. En efecto, si bien es cierto las horas ocupadas en entretención superan las dedicadas a fines educativos o laborales, no es 
menos cierto que el uso en ambos sentidos es aproximadamente equivalente. Es decir, a mayor número de horas conectado en general, aumenta el número de horas conectado exclusivamente a actividades de educación/laboral como también a las de entretención.

El número de horas según tipo de conexión (entretención o educación/laboral) es un cruce básico para construir una caracterización del tipo de inclusión, más bien pasiva o más activa, pero los datos no son suficientemente aclarativos. Se podría testear la hipótesis que existe un continuo entre las actividades de entretención y las de educación donde la probabilidad de un uso intensivo (en horas) es mayor en el extremo "entretención" y menor en el extremo "educación/laboral". Existe un dato que, empero, desdibuja lo anterior, cual es que la mayoría de los jóvenes reenvía la información que es "interesante" (donde cabe tanto la posibilidad que sea algo entretenido como educativo o laboral) y por tanto retroalimenta la comunicación en las redes sociales entre ellos, en ambas dimensiones, entretención y laboral.

Un dato de interés a propósito de la posibilidad de construir una tipología de usuarios es que las mujeres poseen una mayor dispersión en la variable cantidad de horas dedicadas a la entretención. La variable edad es poco robusta, probablemente porque está intervenida por la etapa de estudio universitario: es decir, al principio se dedica menos tiempo al entretenimiento, debido a que se debe rutinizar la actividad; una vez "acostumbrados" al ritmo universitario de estudio, aumenta la dedicación a actividades de esparcimiento online para, posteriormente, volver a disminuirlas, a causa de la cercanía de egreso y actividades de titulación.

Las habilidades protocolares, como el uso de herramientas básicas de sintaxis de búsqueda en Google es (aparentemente) bajo. Da la impresión que existen escasos conocimientos al respecto o bien, no existe el hábito de usarlos.

La calibración y evaluación de la información existente en internet es adecuada para el trabajo universitario en general. La única excepción es el poco uso de Wikipedia, 
seguramente debido a una mala comprensión de las (probables) advertencias docentes: "no usen SOLO wikipedia". Dos tercios de los estudiantes sólo revisa las primeras 10 entradas de Google al hacer una búsqueda, lo cual genera probablemente poca innovación y el intercambio de las mismas ideas entre ellos.

En términos de habilidades técnicas, los resultados son mixtos. Se destacan algunas deficiencias, como el bajo dominio de Excel y el porcentaje bajo de estudiantes que podrían construir una página web. Incluso el dato que 1 de cada 5 sea incapaz de instalar aplicaciones en su móvil es motivo de intriga.

Se podría decir que se esperaba una mayor proporción de respuestas positivas respecto a la auto-percepción de pertenencia al mundo digital y a la sociedad informacional contemporánea de parte de los estudiantes. Quizás ello podría explicarse debido a una evaluación relativamente certera de parte de los estudiantes en relación a las deficiencias competenciales y de conocimiento que el mismo instrumento les hizo constatar. Sin embargo, también podría deberse a un nivel de apropiación tecnológico en general deficiente. Otra explicación alternativa es que los jóvenes adquieren mayor consciencia de la velocidad del cambio tecnológico en esta etapa de sus vidas, la universitaria, debido a su sobreexposición a información relativa al tema.

\section{Conclusión preliminar}

El trabajo asigna números (en este caso, porcentajes) a los componentes observados (en un grupo de estudiantes universitarios chilenos) de nuestro modelo de Capital Informacional, lo que implica que hemos iniciado el trayecto hacia una medición en sentido estricto. Esta "contabilidad" permite visualizar futuras posibilidades de comparación y medición, pero antes sería necesario definir qué significado puede tener la expresión "Capital Informacional ideal", desde una perspectiva social e históricamente situada, desde nuestro mundo global hoy. 
Sin embargo, podemos adelantar algunas conclusiones preliminares. Así, se postula que la posesión de capital informacional es un componente central en la percepción de parte de los estudiantes respecto a su grado de inclusión digital, lo que se vio reflejado en el trabajo empírico. Además, pensamos que los resultados de nuestra exploración entregan información suficiente para avanzar hacia un diagnóstico de la inclusión digital en Chile. En cualquier caso, es una prueba "fuerte", dado que la muestra está constituida por el segmento social y cultural que - se supone - encarna la naturaleza fundamental de la sociedad informacional contemporánea y será la protagonista del desarrollo de las industrias del conocimiento local.

Nuestro modelo (Tabla 1) se conecta con literatura de ciencia social básica y es compatible con ella, tanto desde un marco teórico general dentro de las ciencias sociales (Bourdieu), así como con un marco emergente centrado en los fenómenos específicos del mundo digital (Hamelink). Por cierto, no obstante, es un modelo en progreso.

Nuestro fundamento es que, si el modus operandi de la sociedad informacional es el uso del mundo digital, entonces las habilidades para hacer un uso adecuado y extenso de las herramientas digitales pueden pronosticar el desempeño de las sociedades nacionales en el futuro inmediato.

El capital informacional se constituye, así, en el corpus principal de ese tránsito hacia el futuro. Dentro de este corpus, existen muchas habilidades y competencias que transversalmente poseen los estudiantes, sin distinción de sexo, edad u origen institucional. Destaca la existencia de ciertas habilidades protocolares y la carencia de algunas habilidades técnicas básicas para el mundo del trabajo. La propuesta de encuesta nos previene de dimensiones y variables de escasa importancia (como la tenencia de móvil). La indagación nos indica que existen claros y oscuros (además de muchos grises) en la descripción que podemos hacer de la realidad educacional universitaria hoy en Chile, el país latinoamericano con un mayor acceso al mundo 
digital, pero donde la posesión de escasas habilidades digitales en varios ámbitos coexiste con una auto-percepción de escasa inclusión en la sociedad de la información y el conocimiento, junto con una auto-percepción débil de ciudadanía digital, más baja que la esperada.

El panorama es heterogéneo y sin duda se requiere aclarar más la potencialidad descriptiva del constructo "Capital informacional", ahondando en el estudio de las habilidades y competencias digitales para comprender mejor el significado de ser digitalmente alfabeto hoy, que es una condición para una inclusión digital efectiva.

\section{Referencias}

Alva de la Selva, A. (2016). El indicador cualitativo "capital informacional": categorías emergentes. Revista de comunicación y ciudadanía digital, 5 (2), 148-176.

Bawden, D. (2008). Origins and concepts of digital literacy. En Lankshear, C., \& Knobel, M., Digital literacies: Concepts, policies and practices (pp. 17-32). New York: Peter Lang.

Biggs, J. (2012). What the student does: teaching for enhanced learning. Higher Education Research \& Development, 31(1), 39-55. doi:10.1080/07294360.2012.642839

Bourdieu, P. (2011). Las estrategias de la reproducción social. Buenos Aires: Siglo $\mathrm{XXI}$.

Burin, D., Coccimiglio, Y., González, F., \& Bulla, J. (2016). Desarrollos recientes sobre habilidades digitales y comprensión lectora en entornos digitales. Psicología, Conocimiento y Sociedad. 6 (1), 191-206. Recuperado de http://revista.psico.edu.uy/index.php/revpsicologia

Castells, M. (1997). La era de la información. Economía, sociedad y cultura. Vol. 1: La sociedad red. Madrid: Alianza Editorial. 
Chinn, M., \& Fairlie, RW. (2004). The Determinants of the Global Digital Divide: A Cross-Country Analysis of Computer and Internet Penetration. Center Discussion $\quad$ Papers, $\quad$ (881). $\quad$ Recuperado de http://www.econ.yale.edu/growth_pdf/cdp881.pdf.

Drabowicz, T. (2017). Social theory of internet use: ¿corroboration or rejection among the digital natives?. Computers \& Education, (105), 57-67.

Drahos P., \& Braithwaite, J. (2003). Information feudalism: ¿who owns the knowledge economy? New York: The New Press.

Ellis, R., \& Goodyear, P. (2010). Students' experiences of e-learning in higher education. The ecology of sustainable innovation. New York \& London: Routledge.

Florida, R. (2002). The rise of the creative class. New York: Basic books.

Hamelink, C. (2001). The ethics of cyberspace. London: Sage.

Hilbert, M. (2013). Technological information inequality as an incessantly moving target: The redistribution of information and communication capacities between 1986 and 2010. Journal of the American Society for Information Science and Technology. Recuperado de martinhilbert.net/TechInfolnequality.pdf.

Lankshear, C., \& Knobel, M. (2008). Digital literacies: Concepts, policies and practices. New York: Peter Lang.

Larusson, J. A., \& White, B. (Eds.). (2014). Learning analytics: from research to practice. New York: Springer.

Marí, V M., \& Sierra, F. (2008). Capital informacional y apropiación social de las nuevas tecnologías. Las redes críticas de empoderamiento local en la Sociedad Europea de la Información. Telos, Revista de Comunicación e Innovación, 74, 126-133. 
Moyo, L. (2009). The digital divide: scarcity, inequality and conflict. En: Creeber, G. \& Martin, R. Digital cultures: Understanding new media. Maidenhead: Open UniversityPress, McGraw-Hill, pp.122-129.

ONU. (2015). Documento final de la reunión de alto nivel de la Asamblea General sobre el examen general de la aplicación de los resultados de la Cumbre Mundial sobre la Sociedad de la Información. Recuperado de http://unctad.org/es/PublicationsLibrary/ares70d125_es.pdf

Paavola, S. \& Hakkarainen, K. (2005). The knowledge creation metaphor: An emergent epistemological approach to learning. Science \& Education, 14, 535-557.

Piketty, T. (2015). El capital en el siglo XXI. FCE: México.

Prieur, A. \& Savage, M. (2013). Emerging forms of cultural capital. Europeansocieties, 15 (2), 246-267.

Van Deursen, A. J. A. M., \& Van Dijk, J. A. G. M. (2014). Modeling traditional literacy, Internet skills and Internet usage: An empirical study. Interacting with Computers, 28 (1). doi: 10.1093/iwc/iwu027

Van Dijk, J. (2005). The deepening divide: inequality in the information age. London: Sage.

Witte, J. \& Mannon, S. (2010). The Internet and social inequalities. New York: Routledge.

Zillien, N. \& Hargittai, E. (2009). Digital Distinction: Status-Specific Types of Internet Usage. Social Science Quarterly, 90 (2), 274-291. 
Formato de citación

Espina, P. y Gibert J. (2017). El capital informacional como condición de la inclusión digital: un análisis exploratorio. Psicología, Conocimiento y Sociedad, 7(2), 35-63. Disponible en: http://revista.psico.edu.uy/ 